\title{
Tabaco y rendimiento cognitivo en pacientes con esquizofrenia: diseño del estudio COGNICO
}

\section{Tobacco and cognitive performance in schizophrenia patients: the design of the COGNICO study}

\author{
Susana Al-Halabí*, Sergio Fernández-Artamendi**, Eva M Díaz-Mesa*, Leticia García-Álvarez*, \\ Gerardo Flórez****, Emilia Martínez Santamaría***, Manuel Arrojo****, Pilar A Saiz*, Paz \\ García- Portilla*, Julio Bobes*. \\ * Centro de Investigación Biomédica en Red de Salud Mental (CIBERSAM), Área de Psiquiatría, Universidad de Oviedo; \\ ** Facultad de Psicología, Universidad de Oviedo; *** Unidad de Conductas Adictivas (UCA), Hospital Santamaría Nai de \\ Ourense; **** Servicio de Psiquiatría. Instituto de Investigación Sanitaria (IDIS). Complejo Hospitalario Universitario de \\ Santiago de Compostela.
}

\section{Resumen}

Las personas con esquizofrenia constituyen una parte sustancial de las personas que todavía fuman. La hipótesis de la automedicación en relación al rendimiento cognitivo mantiene que los pacientes fuman para mejorar su déficit cognitivo basándose en los efectos estimulantes de la nicotina. El objetivo de este artículo es describir la metodología del estudio COGNICO. Estudio cuasiexperimental, observacional, prospectivo, multicéntrico y con seguimiento a 3, 6, 12 y 18 meses. Fue llevado a cabo en tres ciudades del norte de España (Oviedo, Ourense y Santiago de Compostela). Se reclutaron 81 pacientes con esquizofrenia fumadores (edad media de 43,35 años (DT=8,83). 72,8\% varones). Se asignaron a 3 grupos: a) control: pacientes fumadores; b) pacientes que dejan de fumar mediante parches de nicotina; c) pacientes que dejan de fumar mediante vareniclina. Como medida primaria se aplicó la batería neuropsicológica MATRICS. Además, se llevó a cabo una evaluación comprehensiva de los pacientes, que incluía el número de cigarrillos por día, la dependencia física y psicológica a la nicotina y el CO expirado. También se realizó una evaluación clínica general (PANSS, HDRS, ICG, C-SSRS) así como un seguimiento de las medidas antropométricas y los signos vitales. Se pretende identificar la relación entre el patrón de consumo de tabaco y el rendimiento cognitivo mediante la comparación de las puntuaciones en la batería neuropsicológica MATRICS durante los períodos de seguimiento.

Palabras clave: Tabaco; esquizofrenia; rendimiento cognitivo; vareniclina; parches de nicotina.

\section{Abstract}

People with schizophrenia constitute a substantial part of the people who still smoke. Regarding cognitive performance, the self-medication hypothesis states that patients smoke to improve their cognitive deficits based on the stimulating effects of nicotine. The aim of this paper is to describe in detail the methodology used in the COGNICO study. A quasi-experimental, observational, prospective, multicenter study with follow-ups over 18 months was conducted in three cities in northern Spain (Oviedo, Ourense and Santiago de Compostela). A total of 81 outpatient smokers with schizophrenia were recruited with a mean age 43.35 years $(\mathrm{SD}=8.83), 72.8 \%$ of them male. They were assigned to 3 groups: a) control group (smokers); b) patients who quit smoking using nicotine patches; c) patients who quit smoking with Varenicline. The MATRICS neuropsychological battery was applied as a primary measure. In addition, a comprehensive assessment of patients was performed, including the number of cigarettes per day, physical and psychological dependence on nicotine and CO expired. Clinical evaluation (PANSS, HDRS, CGI, C-SSRS), anthropometric measurements and vital signs assessment was also performed. The aim is to identify the relationship between the pattern of tobacco use and cognitive performance by comparing scores on the neuropsychological battery MATRICS during the follow-up periods (3, 6,12 and 18months). The importance of this study lies in addressing a topical issue often ignored by clinicians: the unacceptably high rates of tobacco use in patients with severe mental disorders

Keywords: Tobacco; schizophrenia; cognitive performance; Varenicline; nicotine patches. 


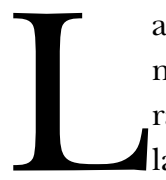

a prevalencia del consumo de tabaco está disminuyendo progresivamente en la población general. Sin embargo, este descenso no se observa en las personas con trastornos mentales graves como la esquizofrenia (García-Portilla et al., 2014). De hecho, estos pacientes constituyen una parte sustancial de las personas que todavía fuman (Lancet, 2013) con tasas que son entre 2 y 4 veces mayores que las de la población general (Lising-Enriquez y George, 2009), o - según algunas publicaciones muy recientes - incluso cinco veces mayor (Beck, Baker y Todd, 2015). En España, la tasa de prevalencia de fumar cigarrillos en los pacientes con esquizofrenia es del 54,5\% (Bobes, Arango, García-García y Rejas, 2010). Estas tasas son prácticamente el doble de la población general española, cuya prevalencia se estima en un $24,1 \%$ (Encuesta Nacional de Salud, 2011/12).

Actualmente es difícil leer una publicación científica acerca del rendimiento cognitivo en los pacientes con trastornos psicóticos que consumen tabaco sin que se aborden aspectos etiopatogénicos de este tipo de consumo o sin que se haga referencia a una posible explicación causal del origen del trastorno adictivo en este tipo de pacientes (Burda et al., 2010; Dervaux y Laquelille, 2008; Dolam et al., 2004; Sacco et al., 2005). Esta elevada prevalencia ha sido registrada en diferentes países y culturas, lo que sugiere que un hipotético factor biológico podría ser el responsable de que estos pacientes sean más propensos a fumar (De Leon, Díaz, Aguilar, Jurado y Gurpequi, 2006). La hipótesis de la automedicación supone un intento de aclarar ese posible factor mediador (Segarra et al., 2010).

Por un lado, numerosas publicaciones defienden la idea de que las personas con esquizofrenia fuman para reducir los efectos adversos de la medicación antipsicótica. De hecho, varios estudios han encontrado que estos pacientes fumadores presentan una menor prevalencia y gravedad de síntomas extrapiramidales en comparación con los pacientes que no fuman (Carrillo et al., 2003; De Leon et al., 2006; Dervaux y Laquelille, 2008). No obstante, existe una gran controversia acerca de este aspecto ya que estos resultados no siempre han sido consistentes (De Leon et al., 2006). Además, parece ser que el intento de alivio de los efectos negativos del tratamiento no podría explicar por sí mismo la alta prevalencia del consumo de tabaco, dado que ésta es similar entre los pacientes crónicos y los que manifestaron un primer episodio psicótico. Los estudios de Beratis, Katrivanou y Gourzis (2001) y Kelly y McCreadie (1999) señalan que el $86-90 \%$ de los pacientes fumadores comenzaron a hacerlo antes de recibir el diagnóstico. Weiser et al. (2004) indican que aquellas personas que están en riesgo de desarrollar una esquizofrenia también presentan factores de riesgo para empezar a fumar.

Por lo tanto, algunos autores sostienen que el factor que está mediando entre el consumo de tabaco y la presencia de un trastorno psicótico debería ser una característica inhe- rente al trastorno que constituya un síntoma premórbido. Y este podría ser el déficit cognitivo, que en la actualidad se presenta con una característica nuclear del trastorno psicótico previa a su manifestación abierta (Andreou et al., 2015; Green y Harvey, 2014; Segarra et al., 2010).

La hipótesis de la automedicación en relación al rendimiento cognitivo mantiene que los pacientes fuman para mejorar sus déficits cognitivos basándose en los efectos estimulantes de la nicotina, que mejoraría la memoria de trabajo visuoespacial y el déficit atencional de estas personas (Depatie et al., 2002; Harris et al., 2004; Jacobsen et al., 2004; Sacco et al., 2005), así como los déficits en el procesamiento sensorial (Leonard y Adams, 2006). No obstante, a este respecto también existen resultados contradictorios, ya que tales beneficios no se han encontrado en otros estudios (Harris et al., 2004; Sacco et al., 2005), y tampoco se han encontrado esos efectos positivos en otros dominios cognitivos como la producción de lenguaje o las funciones ejecutivas (Harris et al., 2004, Sacco et al., 2005; Smith et al., 2006). En nuestro país, en un estudio publicado por Segarra et al. (2010) y llevado a cabo con pacientes con un primer episodio psicótico, se encontró que los fumadores obtuvieron mejores puntuaciones en las tareas de atención y memoria de trabajo después de la primera estabilización de los síntomas clínicos, mientras que los no fumadores obtuvieron mayores ganancias en sus puntuaciones durante el mismo período, de tal manera que, tras un año de tratamiento, ambos grupos desempeñaban por igual las tareas de atención y memoria de trabajo.

En todo caso, los efectos beneficiosos de la nicotina no justificarían el mantenimiento del nocivo hábito tabáquico, ya que el tabaco se asocia con más de 4000 toxinas y de 60 sustancias cancerígenas. Es por esto que en los últimos años algunos autores ya proponen el uso de nicotina (Levin y Rezvani, 2002; Piñeiro et al., 2014) como una vía para modificar la función cognitiva dañada en los pacientes (Smith et al., 2006; Barr et al., 2008).

Por todo lo anteriormente señalado, creemos que un mayor conocimiento sobre el papel que desempeña el consumo de tabaco en el rendimiento cognitivo de los pacientes con esquizofrenia puede contribuir a aclarar los interrogantes que aún existen respecto de esta cuestión y abrir nuevas vías de tratamiento para los déficits neuropsicológicos de estos pacientes basándose en los mecanismos neuronales receptores de nicotina (Levin y Rezvani, 2006). Estos mecanismos han sido identificados como un objetivo terapéutico por el programa MATRICS (Measurement And Treatment Research to Improve Cognition in Schizophrenia) del NIMH ( $N a$ tional Institute on Mental Health), que ha dado lugar a una batería neuropsicológica consensuada para el estudio de la cognición en la esquizofrenia a través de una amplia evaluación científica de medidas (Nuechterlein et al., 2008).

Así, el fin de este artículo es describir la metodología del estudio COGNICO, cuyo objetivo principal es identificar la 
relación entre nicotina y rendimiento cognitivo en pacientes con esquizofrenia mediante la comparación de las puntuaciones en la batería neuropsicológica MATRICS durante un período de seguimiento de 18 meses.

\section{Método}

\section{Diseño del estudio}

Estudio cuasiexperimental, observacional, prospectivo, multicéntrico y con seguimiento a 3, 6, 12 y 18 meses. Fue llevado a cabo en tres ciudades del norte de España (Oviedo, Ourense y Santiago de Compostela) entre 2012 y 2015. La muestra se reclutó en 2 centros de salud mental de Oviedo (CSM Corredoria y CSM La Ería), el Hospital Psiquiátrico de Conxo en Santiago de Compostela y la Unidad de Conductas Adictivas del Complejo Hospitalario de Ourense. El estudio cuenta con tres grupos de participantes:

a. Pacientes con esquizofrenia fumadores.

b.Pacientes con esquizofrenia que dejan de fumar en el momento de iniciarse el estudio (después de la evaluación basal) mediante un tratamiento sustitutivo de la nicotina: parches de nicotina.

c. Pacientes con esquizofrenia que dejan de fumar en el momento de iniciarse el estudio (después de la evaluación basal) y lo hacen mediante métodos que no incluyen el uso de sustitutivos de la nicotina: vareniclina.
Este estudio fue aprobado por el Comité Ético de Investigación Clínica Regional del Principado de Asturias. Todos los participantes del estudio firmaron el consentimiento informado.

\section{Participantes}

Los participantes en el estudio son pacientes con diagnóstico de esquizofrenia bajo tratamiento ambulatorio de mantenimiento. El objetivo inicial de reclutamiento de pacientes para este estudio fue de 20 sujetos por cada grupo de estudio $(n=60)$. Finalmente, un total de 81 pacientes participaron en el estudio con una media de edad de 43,35 años $(\mathrm{DT}=8,83)$. El 72,8\% fueron varones $(\mathrm{n}=59)$. De ellos, 25 pacientes $(30,9 \%)$ fueron asignados al grupo control, $32(39,5 \%)$ al grupo de parches de nicotina y $24(29,6 \%)$ al grupo de vareniclina. El consumo medio de cigarrillos por día en la evaluación basal fue el siguiente: grupo control $=29,76(\mathrm{DT}=13,13)$; grupo parches de nicotina $=26,81$ $(\mathrm{DT}=11,85)$; grupo vareniclina $=27,63(\mathrm{DT}=12,13)$.

La selección de los pacientes se realizó entre aquellos que habían manifestado su deseo de dejar de fumar, o aquellos pacientes fumadores que quisieron colaborar.

Los criterios de inclusión en el estudio fueron: (1) diagnóstico de esquizofrenia según criterios CIE-10 para investigación y que, a juicio del clínico, hubiesen estado estables clínicamente al menos en los 6 meses previos (sin hospita-

Tabla 1. Áreas e instrumentos de evaluación del estudio COGNICO

Área de evaluación Instrumentos de evaluación / Parámetros biológicos

\begin{tabular}{|c|c|c|}
\hline \multirow[t]{3}{*}{ Consumo de tabaco } & \multirow[t]{2}{*}{ Patrón de consumo } & Cigarrillos por día (CPD). \\
\hline & & Nivel de Monóxido de Carbono (CO) expirado. \\
\hline & Dependencia nicotínica & $\begin{array}{l}\text { Test Fagerström de Dependencia a la Nicotina (FTND). } \\
\text { Cuestionario Glover-Nilsson de Dependencia Psicológica (TGN). }\end{array}$ \\
\hline \multirow[t]{2}{*}{ Otras sustancias } & Cafeína & Consumo diario \\
\hline & Otras & Cualquier consumo. \\
\hline \multirow{3}{*}{ Psicopatología } & Depresión & Escala de Depresión de Hamilton (HDRS). \\
\hline & Tentativas de Suicidio & Escala Columbia de Gravedad del Suicidio (C-SSRS). \\
\hline & Gravedad & Escala de Impresión Clínica Global: Gravedad (ICG-G) y Cambio (ICG-C). \\
\hline \multirow[t]{2}{*}{ Evaluación biológica } & Antropometría & Peso, estatura, IMC, perímetro abdominal. \\
\hline & Signos vitales & Presión arterial, pulso. \\
\hline
\end{tabular}


lizaciones o reagudizaciones sintomatológicas significativas que hayan requerido una intensificación del nivel de cuidados psiquiátricos) y que se encuentren en tratamiento de mantenimiento; (2) fumadores actuales de al menos 10 pitillos/día durante el último año sin un periodo de abstinencia mayor de 1 mes en ese último año; (3) edad comprendida entre los 18 y 65 años; (4) no ideación suicida actual y (5) consentimiento informado firmado. Los pacientes fueron excluidos si cumplían alguno de los siguientes criterios: (1) puntuaciones superiores a 70 puntos en la PANSS o superiores a 20 puntos en la Escala de Depresión de Hamilton (HDRS), (2) presencia de comportamientos o ideación suicida grave en los 6 meses previos, o (3) historia de daño cerebral orgánico incluyendo epilepsia, tumores, traumatismos craneoencefálicos con deterioro cognitivo significativo.

\section{Variables e instrumentos de evaluación}

Todas las evaluaciones (ver Tabla 1) fueron llevadas a cabo por psicólogos y psiquiatras debidamente entrenados para tal fin y se realizaron durante todos los seguimientos (excepto los datos sociodemográficos y clínicos que sólo se recogieron durante la evaluación basal).

\section{Datos sociodemográficos y clínicos}

Se recogieron los datos de edad, sexo, estado civil, nivel de estudios, profesión y situación laboral. Los datos clínicos registrados fueron los siguientes: diagnóstico principal (realizado por un psiquiatra), diagnóstico secundario, duración del trastorno, primer episodio, intentos previos de suicidio y tratamiento psicofarmacológico actual.

\section{Medidas antropométricas y signos vitales}

Se midió la altura, el peso (sin chaquetas o abrigos y sin zapatos) y el perímetro abdominal. Se calculó el índice de masa corporal (IMC). Los signos vitales que se recogieron fueron el pulso y la presión arterial, ambos medidos después de unos minutos de reposo del paciente.

\section{Patrón de consumo de tabaco}

El patrón de consumo de tabaco se registró mediante los siguientes parámetros: el número de cigarrillos fumados por día, nivel de monóxido de carbono (CO) expirado, y dependencia física y psicológica a la nicotina. Se evaluó la posible presencia de síntomas de abstinencia a la nicotina mediante los criterios del DSM-IV-TR.

- Número de Cigarrillos por Día (CPD): el número de CPD se puede considerar una medida válida de la dependencia nicotínica. Dado que no existe ningún consenso a la hora de clasificar a los fumadores en niveles bajos y altos de consumo, en este estudio se decidió clasificarlos en los siguientes grupos basándonos en los criterios de García-Portilla et al., 2014: consumo leve (CPD < 10 cigarrillos $)$, moderado $(\mathrm{CPD}=11-20$ cigarillos $)$, y alto $(\mathrm{CPD}=>20$ cigarrillos $)$
- Nivel de Monóximo de Carbono Expirado (CO): se evaluó utilizando un piCOsimpleTM Smokerlizer ${ }^{\circledR}$. El punto de corte para el criterio de "fumadores actuales" fue de 6ppm (siguiendo instrucciones del fabricante). Las mediciones de CO se realizaron siempre a primeras horas de la mañana.

- Test Fagerström de Dependencia a la Nicotina (TFDN) (Becoña y Vazquez, 1998). Incluye un total de 6 ítems que evalúan el grado de dependencia fisiológica. La puntuación total oscila entre 0 y 10 puntos, y categoriza a los participantes en dependencia leve (0-3), moderada (4-7) y grave (8-10).

- Test de Glover-Nilsson (TGN) (Nerin et al., 2005). Está compuesto por 11 ítems que evalúan la dependencia psicológica y conductual a la nicotina. A partir de sus puntuaciones se clasifica a los participantes en cuatro niveles: dependencia leve (0-11), moderada (12-22), fuerte (23-33) y muy fuerte (34-44).

\section{Consumo de sustancias}

Se evaluó el consumo de cafeína, alcohol, cannabis, cocaína y otras sustancias.

\section{Evaluación neuropsicológica}

Para la evaluación del funcionamiento neuropsicológico se utilizó la batería de consenso MATRICS (Measurement and Treatment Research to Improve Cognition in Schizofrenia) (Nuechterlein et al., 2008). Véase Tabla 1 para una descripción más detallada.

\section{Evaluación psicopatológica}

Los instrumentos de evaluación clínica incluían las siguientes escalas:

- Escala del Síndrome Positivo y Negativo de la Esquizofrenia (PANSS) (Peralta y Cuesta, 1994): evalúa la gravedad de los síntomas (positivos, negativos y psicopatología general) de la esquizofrenia. Cada ítem tiene una puntuación de 0 a 7 (la puntuación total oscila entre 30 y 120). Puntuaciones más altas indican mayor gravedad de los síntomas.

- Escala Hamilton para la Depresión (HDRS) (Bobes et al., 2003): consta de 17 ítems que evalúan el perfil sintomatológico y la gravedad del cuadro depresivo. Proporciona una puntuación global que oscila entre 0 y 52 puntos. A mayor puntuación, mayor gravedad.

- Escala de Columbia de Valoración de la Gravedad del Suicidio (C-SSRS) (Al-Halabí et al., en prensa): entrevista semiestructurada que evalúa la tanto la ideación como la conducta suicidas. No existe una puntuación global ni puntos de corte definidos.

- Impresión Clínica Global en sus versiones de Gravedad y Cambio (ICG-G e ICG-C) (Guy, 1976): evalúa la gravedad total del trastorno (esquizofrenia, en este caso). 
Cada ítem se puntúa en una escala Likert de 7 puntos (desde normal a extremadamente enfermo).

\section{Tratamiento para dejar de fumar}

La elección del método para dejar de fumar fue realizada en función de la disponibilidad del tratamiento, las experiencias previas del paciente y sus preferencias, y el juicio clínico. Los tratamientos farmacológicos utilizados en este estudio fueron aquellos aprobados y considerados como primera opción por el Servicio de Salud Pública de Estado Unidos (Guideline Update Panel, 2008). Asimismo, la Asociación Europea de Psiquiatría (EPA) incluye los parches de nicotina y la vareniclina dentro de los tratamientos farmacológicos para dejar de fumar en todos los pacientes con algún tipo de trastorno mental (Rüther et al., 2014). Las dosificaciones se realizaron siguiendo el protocolo habitual (García-Portilla et al., 2014). En caso de descompensación psicopatológica o efectos adversos importantes estaba previsto suspender el tratamiento y excluir al participante del estudio. Adicionalmente, todos los pacientes que iniciaron el tratamiento para dejar de fumar recibieron consejo dietético, técnicas de control estimular (eliminar estímulos elicitadores de fumar) y sugerencias para la adquisición de hábitos saludables.

\section{Plan Estadístico}

Se obtendrán los estadísticos descriptivos para todas las variables clínicas y sociodemográficas y se analizarán las posibles diferencias previas entre los grupos. Como medida principal se examinarán los cambios en las puntuaciones medias de la batería MATRICS en cada uno de los momentos de evaluación (3, 6, 12 y 18 meses). Se analizará si el rendimiento cognitivo es significativamente diferente entre los pacientes fumadores y los que dejan de fumar. A su vez, se analizará si hay diferencias entre los que dejan de fumar mediante sustitutivos de la nicotina y los que lo hacen mediante otros métodos. Igualmente, como resultados secundarios, se examinarán los cambios en las puntuaciones medias de las escalas de evaluación clínica (PANSS, HDRS, C-SSRS, ICG). Previamente a los análisis estadísticos, se examinarán las características de la distribución de la muestra y la presencia de outliers. Se considerará un nivel de significación estadística bilateral con un nivel de confianza del $95 \%$.

\section{Discusión}

Este artículo describe con detalle la metodología diseñada y utilizada en el estudio COGNICO, cuyo objetivo es identificar la relación entre nicotina y rendimiento cognitivo en pacientes con esquizofrenia. Para ello, se llevará a cabo la comparación de las puntuaciones obtenidas por los participantes en la batería neuropsicológica MATRICS durante un período de seguimiento de 3, 6, 12 y 18 meses.

La importancia de este estudio radica en que aborda un tema que con frecuencia ha sido ignorado por los profesio- nales de la salud mental: la tasa de consumo de tabaco entre los pacientes con esquizofrenia es alarmantemente alta (Bachiller et al., 2015; García-Portilla et al., 2014). A este respecto, la Asociación Europea de Psiquiatría (EPA) señala la necesidad de llevar a cabo intervenciones en este área, así como conocer el impacto que tiene la dependencia del tabaco en nuestros pacientes (Rüther et al., 2013. Precisamente este estudio se enmarca en esa línea de investigación. A pesar de todo lo anterior, sólo unos pocos estudios han examinado la eficacia y seguridad de los programas para dejar de fumar en pacientes con trastornos mentales (García-Portilla et al., 2014).

Uno de los últimos trabajos publicados sobre este tema (Asahre, Falcones y Lerman, 2014), lejos de arrojar luz, deja constancia de que estamos ante un tema complejo que aún no ha sido resuelto. Estos autores señalan que el abandono de nicotina está asociado con déficits neurocognitivos que incluyen la atención sostenida, la memoria de trabajo y la respuesta de inhibición. Y añaden: "lo que está claro tras nuestra revisión es que los efectos de la abstinencia de la nicotina sobre la función cognitiva son mucho más complejos de lo que se teorizó inicialmente”. Boggs, Carlson, Cortes-Briones, Krystal y D’Souza (2014) señalan que es necesario un mayor conocimiento del sistema nicotínico para determinar si estamos ante una nueva diana terapéutica que permita mejorar el rendimiento cognitivo.

Uno de los puntos fuertes de este estudio es su validez externa y la generalidad de los resultados. Los criterios de inclusión y exclusión utilizados nos han permitido reclutar pacientes "reales". Nuestro objetivo es estudiar qué ocurre con nuestros pacientes cuando dejan de fumar, sin necesidad de utilizar métodos sofisticados de laboratorio para evaluar los $m g$ de nicotina $u$ otras condiciones experimentales poco factibles en la práctica cotidiana. Otro aspecto positivo es el tamaño de la muestra. Aunque 81 pacientes no es un número muy ambicioso, la mayoría de los estudios publicados incluyen un tamaño muestral inferior (García-Portilla et al., 2014). Además de lo anteriormente señalado, nos gustaría destacar que se llevó a cabo una evaluación comprehensiva de los pacientes, ya que no sólo se ha aplicado la MATRICS o registrado el número de cigarrillos, sino que se han tenido en cuenta otros aspectos del patrón de consumo de tabaco como la dependencia física y psicológica y el CO. También se ha realizado una evaluación clínica general, incluyendo ideación suicida, con instrumentos fiables y válidos, así como un seguimiento de las medidas antropométricas y los signos vitales. Todo ello añade valor a este estudio.

No obstante, existen limitaciones. La más importante es la ausencia de un grupo control de pacientes que, no siendo fumadores, comiencen a fumar justo después de la evaluación basal del estudio. Las evidentes dificultades para conseguir este tipo de muestra son de carácter empírico y ético. Asimismo, otra limitación sería el hecho de que el tratamiento para dejar de fumar sea de carácter naturalístico, no 
controlado. Sin embargo este tipo de limitaciones, inherentes a los estudios abiertos, garantizan una mayor semejanza con la práctica clínica cotidiana.

\section{Reconocimientos}

Este estudio ha sido financiado por el Instituto de Salud Carlos III (Referencia: PI11/01891), cofinanciado por el Fondo Europeo de Desarrollo Regional (FEDER) (Unión Europea. "Una forma de hacer Europa") y gestionado por el Centro de Investigación Biomédica en Red de Salud Mental, CIBERSAM.

\section{Conflicto de intereses}

No existe conflicto de intereses.

\section{Referencias}

Al-Halabí, S., Sáiz, P.A., Burón, P., Garrido, M., Benabarre, A., Jiménez, E., ... Bobes, J. (en prensa). Validation of a Spanish version of the Columbia-Suicide Severity Rating Scale (C-SSRS). Revista de Psiquiatría y Salud Mental.

Andreou, C., Schneider, B.C., Balzan, R., Luedecke, D., Roesch-Ely, D., Moritz, S. (2015). Neurocognitive deficits are relevant for the jumping-to-conclusions bias, but not for delusions: A longitudinal study. Schizophrenia Research: Cognition, 2, 8-11. doi:10.1016/j. scog.2015.02.001.

Ashare, R.L., Falcones, M., y Lerman, C. (2014). Cognitive function during nicotine withdrawal: Implications for nicotine dependence treatment. Neuropharmacology, 76, 581-591. doi:10.1016/j.neuropharm.2013.04.034.

Bachiller, D., Grau-López, L., Barral, C., Daigre, C., Alberich, C., Rodríguez-Cintas, L., ... Roncero, C. (2015). Grupo motivacional en unidad hospitalaria desintoxicación, su influencia en mantenimiento de la abstinencia y retención al tratamiento tras alta. Adicciones, 27, 09-118.

Barr, R.S., Culhane, M.A., Jubelt, L.E., Mufti, R.S., Dyer, M.A., Weiss, A.P., ... Evins, A.E. (2008). The effects of transdermal nicotine on cognition in nonsmokers with schizophrenia and nonpsychiatric controls. Neuropsychopharmacology, 33, 480-490.

Beck, A.K., Baker, A.L., y Todd, J. (2015). Smoking in schizophrenia: cognitive impact of nicotine and relationship to smoking motivators. Schizophrenia Research: Cognition, 2, 26-32. doi:10.1016/j.scog.2014.12.001.

Becona, E., y Vazquez, F.L. (1998). The Fagerstrom test for nicotine dependence in a Spanish sample. Psychological Report, 83, 1455-1458.

Beratis, S., Katrivanou, A., y Gourzis, P. (2001) Facotrs affecting smoking in schizophrenia. Comprehensive Psychiatry, 42, 393-402.
Bobes, J., Arango, C., García-García, M., y Rejas, J. (2010). Healthy lifestyle habits and 10-year cardiovascular risk in schizophrenia spectrum disorders: an analysis of the impact of smoking tobacco in the CLAMORS schizophrenia cohort. Schizophrenia Research, 119, 101-109. doi: 10.1016/j.schres.2010.02.1030.

Bobes, J., Bulbena, A., Luque, A., Dal-Ré, R., Ballesteros, J., e Ibarra, N. (2003). A comparative psychometric study of the Spanish versions with 6, 17, and 21 items of the Hamilton Depression Rating Scale. Medicina Clinica, 120, 693-700.

Boggs, D.L., Carlson, J., Cortes-Briones, J., Krystal, J.H., y D'Souza, D.C. (2014). Going up in smoke? A review of nAChRs-based treatment strategies for improving cognition in schizophrenia. Current Pharmaceutical Design, 20, 5077-5792.

Burda, K., Czubak, A., Nowakowska, E., Kus, K., Metelska, J., y Nowakowska, A. (2010). Interactions of nicotine and drugs used in the treatment of mental illnesses with respect to cognitive functions. Arzneimittelforschung, 60, 527-543. doi: 10.1055/s-0031-1296322.

Carrillo, J.A., Herraiz, A.G., Ramos, S.I., Gervaisni, G., Vizcaíno, S., y Benítez, J. (2003). Role of the somokinginduced cytochrome P450 (CYP) $1^{\text {a }} 2$ and polymorphic CYP2D6 in steady-state concentration of olanzapine. Journal of Clinical Psychopharmacology, 23, 119-127.

Depatie, L., O’Driscoll, G.A., Holahan. A.L., Atikson, V., Thayundayil, J.X., Kin, N.N., y Lal, S., (2002). Nicotine and behavioral markers of risk for schizophrenia: a double-blind, placebo-controlled, cross-over study. Neuropsychopharmacology, 27, 1056-1070.

De Leon, J., Díaz, F.J., Aguilar, M.C., Jurado, D., y Gurpequi, M. (2006). Does somoking reduce akathisia? Testing a narrow version of the self-medication hypothesis. Schizophrenia Research, 86, 256-268.

Dervaux, A., y Laquelille, X. (2008). Tobacco and schizophrenia: epidemiological and clinical features. Encephale, 34, 299-305. doi: 10.1016/j.encep.2007.04.003.

Dolam, S.L., Sacco, K.A., Termine, A., Seyal, A.A., Dudas, M.M., Vessicchio, J.C., ... George, T.P. (2004). Neuropsychological deficits are associated with smoking cessation treatment failure in patients with schizophrenia. Schizophrenia Research, 70, 263-275.

Encuesta Nacional de salud 2011/2012. Recuperado con fecha 28 de octubre de 2015 de http://www.msssi.gob. es/estadEstudios/estadisticas/encuestaNacional/encuesta2011.htm.

García-Portilla, M.P., García-Álvarez, L., Saiz, P.A., DíazMesa, E., Galván G., Sarramea, F., ... Bobes, J. (2014). Effectiveness of a multi-component smoking cessation support programme (McSCSP) for patients with severe mental disorders: study design. International Journal of Environmental Research and Public Health, 11, 373-389. 
Green, M.F., y Harvey, P.D. (2014). Cognition in schizophrenia: Past, present, and future. Schizophrenia Research: Cognition, 1, 1-9. doi:10.1016/j.scog.2014.02.001.

Guideline Update Panel, Liaisons, and Staff (2008). Treating tobacco use and dependence: 2008 update U.S. Public Health Service Clinical Practice Guideline executive summary. Respiratoty Care, 53, 1217-1222.

Guy, W. (1976). ECDEU Assessment Manual for Psychopharmacology - Revised. Rockville, MD: U.S. Department of Health, Education and Welfare, Public Health Service, Alcohol, Drug Abuse and Mental Health Administration, NIMH.

Harris, J.G., Kongs, S., Allensworth, D., Martin, L., Tregellas, J., Sullivan B., y Freedman, R. (2004). Effects of nicotine on cognitive deficit in schizophrenia. Neuropsychofarmacology, 29, 1378-1385.

Jacobsen, L.K., D’Souza, D.C., Mencl, W.E., Pugh, K.R., Skudlarski, P., y Krystal, J.H. (2004). Nicotine effects on brain function and functional connectivity in schizophrenia. Biological Psychiatry, 55, 850-858.

Kelly, C., y McCreadie, R.G. (1999) Smoking habits, currents symptoms, and premorbid characteristics of schizophrenic patients in Nitthsdale, Scotland. American Journal of Psychiatry, 156, 1751-1757.

Lancet, E. (2013) Smoke alarm: Mental illness and tobacco. Lancet, 381, 1071.

Leonard. S., y Adams, C.E. (2006). Smoking cessation and schizophrenia. American Journal of Psychiatry, 163, 1877.

Levin, E.D., y Rezvani, A.H. (2002). Nicotinic treatment for cognitive dysfunction. Current Drug Targets. CNS and Neurological Disorders, 4, 423-431.

Levin, E.D., y Rezvani, A.H. (2006). Nicotinic-antipsychotic drug interactions and cognitive function. Experientia Supplementum, 98, 125-205.

Lising-Enriquez, K., y George, T.P. (2009). Treatment of comorbid tobacco use in people with serious mental illness. Journal of Psychiatry and Neuroscience, 34, E1-E2.

Nerin, I., Crucelaegui, A., Novella, P., Beamonte, A., Sobradiel, N., Bernal, V., Gargallo, P. (2005) Assessment of behavioral dependence with the glover-nilsson test in smoking cessation treatment. Archivos de Bronconeumología, 41, 493-498.

Nuechterlein, K.H., Green, M.F., Kern, R.S., Baade, L.E., Barch, D.M., Cohen, J.D., ... Marder, S.R. (2008) The MATRICS Consensus Cognitive Battery, part 1: test selection, reliability, and validity. American Journal of Psychiatry, 165, 203-213.

Peralta, V., y Cuesta, M.J. (1994). Psychometric properties of the positive and negative syndrome scale (PANSS) in schizophrenia. Psychiatric Research, 53, 31-40.

Piñeiro,B., López-Durán, A., Fernández del Río, E., Martínez, U., Brandon, T.H., y Becoña, E. (2014). Craving and nicotine withdrawal in a Spanish smoking cessation sample Craving y abstinencia de la nicotina en fumado- res españoles en un tratamiento para dejar de fumar. Adicciones, 26, 230-237.

Rüther, T., Bobes, J., De Hert, M., Svensson, T., Mann, K., Batra, A., ... Möller, H.J. (2014). EPA-Position statement on smoking and strategies for smoking cessation in people with mental illness. European Psychiatry, 29, 65-82. doi:10.1016/J.EURPSY.2013.11.002.

Sacco, K.A., Termine, A., Seyal, A., Dudas, M.M., Vessichio, J.C., Krishnan-Sarin, S., y George, T.P. (2005). Effects of cigarette smoking on spatial working memory and attentional deficits in schizophrenia: involvement of nicotinic receptor mechanisms. Archives of General Psychiatry, 62, 649-659.

Segarra, R., Zabala, A., Eguíluz, J.I., Ojeda, N., Elizagarate, E., Sánchez, P., ... Gutiérrez, M. (2010). Cognitive performance and smoking in first-episode psychosis: the self-medication hypothesis. European Archives of Psychiatry Clinical Neuroscience, 261, 241-250. doi:10.1007/ s00406-010-0146-6.

Smith, R. C., Warner-Cohen, J., Matute, M., Butler, E., Kelly, E., Vaidhyanathaswamy, S., y Khan, A. (2006). Effects of nicotine nasal spray on cognitive function in schizophrenia. Neuropsychopharmacology, 31, 637-643.

Weiser, M., Reichenberg, A., Grotto, I., Yasvitzky, B., Rabinowitz, J., Lubin, G., ... Davidson, M. (2004). Higher rates of cigarette smoking in male adolescents before the onset of schizophrenia: a historical-prospective cohort study. American Journal of Psychiatry, 161, 1219-1223. 